\title{
A STRONG OPERATOR TOPOLOGY ADIABATIC THEOREM
}

\author{
ALEXANDER ELGART AND JEFFREY H. SCHENKER
}

\begin{abstract}
We prove an adiabatic theorem for the evolution of spectral data under a weak additive perturbation in the context of a system without an intrinsic time scale. For continuous functions of the unperturbed Hamiltonian the convergence is in norm while for a larger class functions, including the spectral projections associated to embedded eigenvalues, the convergence is in the strong operator topology.
\end{abstract}

\section{INTRODUCTION}

The aim of this paper is to give a slightly new perspective on adiabatic theorems related to systems without intrinsic time scales. We consider convergence in the strong operator topology and hope to convince the reader that this is a natural setting for adiabatic theorems when there is no intrinsic notion of "slowness."

The adiabatic theorem of quantum mechanics describes the behavior of a nonautonomous system driven by means of slowly altered external field. An illustrative example is the case of a spin-1/2 particle (two level system) coupled to a rotating magnetic field of constant amplitude. The evolution of this system is generated by the time dependent Hamiltonian $H(t):=\vec{\sigma} \cdot \vec{B}(t)$, where $\left\{\sigma_{i}\right\}$ are the Pauli matrices and $\vec{B}$ is the rotating magnetic field. There are two time scales here: the inverse of the rate at which magnetic field is changing, $t_{1}:=|B| /|\dot{B}|$, and the intrinsic time scale of the two level system, $t_{2}:=1 /|B|$, which is linked to the gap between the energy levels of the instantaneous Hamiltonian. It is natural to say that the system changes "slowly" if the ratio $t_{2} / t_{1}$ is small. Adiabatic theory [1] implies in this context that if initially the system is in a stationary state - for instance, with the spin parallel to the magnetic field $\vec{B}(0)$ - then it will stay close to an instantaneous stationary state - i.e., parallel to the direction of $\vec{B}(t)$. "Close" here means that the transition amplitude to the second stationary state - anti-parallel to $\vec{B}(t)$ - is bounded from above by a function of the ratio $t_{2} / t_{1}$ which vanishes at zero.

In general, the subject of quantum adiabatic theory is the unitary evolution which solves an initial value problem (Schrödinger equation) of the form

$$
\left\{\begin{array}{l}
\mathrm{i} \dot{U}_{\tau}(t)=H(t / \tau) U_{\tau}(t), \quad t \in[0, \tau] \\
U_{\tau}(0)=\mathbf{1}
\end{array},\right.
$$

with a time dependent self adjoint operator $H(s)$ for $s \in[0,1]$. The parameter $\tau$ is supposed to provide a scale to measure the "slowness" of the system, and adiabatic theory is concerned with the limit $\tau \rightarrow \infty$. Strictly speaking, to determine what is meant by "slow," we need a second time scale coming from the structure of the system - e.g., a spectral gap as in the above example.

Date: February 25, 2002. 
When applicable, the adiabatic theorem states that

$$
\lim _{\tau \rightarrow \infty} U_{\tau}(\tau s) f(H(0)) U_{\tau}^{\dagger}(\tau s)=f(H(s)), \quad s \in[0,1] .
$$

However, to be precise we should indicate in what topology this limit is taken, and this issue is the heart of this work.

As far as we know, to date eq. (1.2) has always been understood in the norm sense. This choice has been well justified since for the systems in question the the meaning of slowness was intrinsic. For instance, if the function $f$ in $(1.2)$ is a projection to a spectral band separated by a finite gap from the rest of the spectrum, then the adiabatic theorem holds in the norm sense [2, 3]. In this case, the inverse of the spectral gap provides an intrinsic time scale.

There are also examples of systems without a spectral gap but nonetheless a clearly defined intrinsic time scale. The first example is the so called level crossing situation: Imagine that two non-degenerate eigenvalues of the instantaneous Hamiltonian cross each other at some time. Although the spectral gap vanishes at the crossing, there is an intrinsic time scale coming from the relative slope of the eigenvalues. There is an adiabatic theorem in this case which holds in the norm sense 揷. Recently this was extended to systems with an infinite number of crossings in finite time [5]. Another example is a system with dense point spectrum perturbed by a finite rank operator, considered in ref. [6]. There, the time scale is related not to the gap between energies (which may be arbitrarily small) but the gap multiplied by the overlap between the corresponding eigenstates coupled through the perturbation. Our final example is a system with an eigenvalue of finite degeneracy at the threshold of, say, continuous spectrum as considered in [7, 8]. Here an intrinsic time scale can be extracted from the Hölder continuity of the continuous part of the spectral measure in the vicinity of the eigenvalue. In all situations above, the adiabatic theorem holds in the norm topology, and, more or less, these examples exhaust the known results on the subject.

The present paper is concerned with the adiabatic theorem for a system without an intrinsic time scale. We are motivated by problems encountered in the analysis of the Quantum Hall Effect (QHE) in which one considers a time dependent perturbation of a system with dense point spectrum. Unlike in ref. [6], the perturbation is not finite rank which has the consequence, as was pointed out to us by Michael Aizenman, that one does not expect the adiabatic theorem to be true in the norm operator topology in that context. A somewhat simpler example of the phenomenon which occurs there is provided by a direct sum of infinitely many non-interacting systems each of which with its own characteristic time scale. Once the adiabatic parameter $\tau$ is larger than the time scale of an individual system, that subsystem is close to the adiabatic limit. However, if the sequence of time-scales is unbounded, there is no notion of slowness which holds for the whole system. We discuss this example in more detail in Section 5 .

We consider in this paper a family of Hamiltonians of the form

$$
H_{\tau}(t / \tau)=H_{o}+\frac{1}{\tau} \Lambda(t / \tau)
$$

where $H_{o}$ and $\Lambda(s)$, for $s \in[0,1]$, are self adjoint operators. The particular form for the time dependence is formulated with the QHE in mind.

${ }^{a}$ There is an extensive literature on adiabatic theory, much of which is not cited here. Several extensive reviews have appeared recently (see 8, 96). 
The usual adiabatic framework involves a Hamiltonian which depends on $\tau$ only through the rescaling of time - see eq. (1.1). The evolution consider here is equivalent, via a unitary transformation, to the solution of (1.1) with $H(s)=$ $V(s) H_{o} V^{\dagger}(s)$, where $\Lambda(s)$ is the generator of $V(s)$ :

$$
\mathrm{i} \dot{V}(s)=\Lambda(s) V(s), \quad V(0)=\mathbf{1} .
$$

In physical literature, this description of the dynamics is referred to as the "interaction picture," and has proved useful in many situations.

We discuss here the limit $\tau \rightarrow \infty$ of a solution, $A_{\tau}(t)=U_{\tau}(t) A(0) U_{\tau}^{\dagger}(t)$, to the associated Heisenberg equation

$$
\mathrm{i} \dot{A}_{\tau}(t)=\left[H_{\tau}(t / \tau), A_{\tau}(t)\right]
$$

when the initial observable is a function of $H_{o}$, i.e., $A(0)=f\left(H_{o}\right)$. Our main result, Theorem 11, states that

$$
U_{\tau}(\tau) f\left(H_{o}\right) U_{\tau}^{\dagger}(\tau) \longrightarrow f\left(H_{o}\right)
$$

for a wide class of functions $f$.

The topology in which eq. 1.6 holds depends on the continuity of $f$ relative to the spectral properties of $H_{o}$ : for continuous functions we obtain norm convergence while for a class of discontinuous functions we obtain strong operator convergence. Let us recall that a family $\tau \mapsto A_{\tau}$ of operators converges to $A$ in the strong operator topology (SOT) if

$$
\lim _{\tau \rightarrow \infty} A_{\tau} \psi=A \psi
$$

for every $\psi \in \mathcal{H}$ and converges in norm if

$$
\lim _{\tau \rightarrow \infty}\left\|A_{\tau}-A\right\|=0
$$

We denote SOT convergence by "SOT-lim $A_{\tau}=A$ ".

The remainder of this paper is organized as follows. Sections 2, 3 and 4 are devoted to the statement and proof of our main result, Theorem 11 in section 2 . In section 5 we describe an example which shows that the norm topology is inadequate when we consider discontinuous functions of $H_{o}$. Finally, in section 6 we present a stronger result which holds when $H_{o}$ has pure point spectrum.

\section{ThE THEOREM AND ALL WE CAN SHOW WITH THE RESOLVENT}

Before we state Theorem 1, let us recall the definition of certain classes of functions $f: \mathbb{R} \rightarrow \mathbb{C}$ :

(1) Let $C_{b}$ denote the bounded continuous functions.

(2) Let $C_{o}$ denote those functions in $C_{b}$ which vanish at $\pm \infty$.

(3) Let $B V$ denote the functions of bounded variation, i.e., functions $f$ for which

$$
\operatorname{Var}(f):=\sup _{n \geq 1} \sup _{x_{o}<\cdots<x_{n} \in \mathbb{R}} \sum_{j=1}^{n}\left|f\left(x_{j}\right)-f\left(x_{j-1}\right)\right|<\infty .
$$

A function in $B V$ can have only countably many points of discontinuity. We direct the reader to [10, Ch. 3] for a detailed discussion of $B V$. 
Theorem 1. Let $H_{o}$ be a self adjoint operator and suppose that the time evolution $U_{\tau}$ satisfies the initial value problem (1.1) with $H_{\tau}(t / \tau)=H_{0}+(1 / \tau) \Lambda(t / \tau)$ where $\Lambda(\cdot)$ is a self adjoint family which is $L^{1}$ in norm: $\int_{0}^{1} \mathrm{~d} s\|\Lambda(s)\|<\infty$. Given a measurable function $f$, consider the statement

$$
\lim _{\tau \rightarrow \infty} W_{\tau}(s) f\left(H_{0}\right) W_{\tau}^{\dagger}(s)=f\left(H_{o}\right), \text { uniformly for } s \in[0,1],
$$

where $W_{\tau}$ is the evolution at scaled time, $W_{\tau}(s)=U_{\tau}(\tau \cdot s)$.

(1) If $f \in C_{o}$ then eq. (2.2) is true in the operator norm topology.

(2) If $f=g+h$ with $g \in C_{b}$ and $h \in B V$ then eq. (2.2) is true in the strong operator topology.

Remarks:

(1) Operators $A_{\tau}(s)$ are said to converge uniformly to $A$ in the strong operator topology if

$$
\lim _{\tau \rightarrow \infty} \sup _{s}\left\|A_{\tau}(s) \psi-A \psi\right\|=0
$$

for every $\psi \in \mathcal{H}$. Uniform norm convergence is defined similarly.

(2) Among the usual operator topologies, i.e., the norm topology as well as the strong and weak operator topologies, the strong operator topology is the strongest in which we can expect an adiabatic limit for discontinuous functions of $H_{o}$. In section 5 we describe an elementary example of a system for which $W_{\tau}(s) f\left(H_{o}\right) W_{\tau}^{\dagger}(s)$ fails to converge in the norm topology.

(3) If the operator $H_{o}$ is unbounded, the distinction between $C_{o}$ and $C_{b}$ is meaningful. Functions in $C_{b}$ may be "discontinuous at infinity" which explains the loss of norm convergence.

(4) Among the functions of bounded variation are the Krönecker delta functions: $\delta_{E}(x)=1$ if $x=E$ and 0 otherwise. Thus we obtain an adiabatic evolution for the spectral projection associated to any eigenvalue - even if it has infinite degeneracy and is embedded in the essential spectrum!

(5) As described above, the standard adiabatic theorems describe the limiting behavior of the Schrödinger evolution for a system having a gap in its spectrum with initial data being a spectral projection onto an energy band. A projection onto a spectral band is a continuous function of $H_{o}$, thus the convergence occurs in the norm topology. In such a setting it is possible to find an explicit bound on the rate of convergence in eq. 2.2 (see, for example, eq. (2.12) and Lemma 3).

(6) Schrödinger equations with a Hamiltonian of the form considered here find direct application in the description of the motion of a quantum particle in a time dependent potential energy. In that case, $H_{o}$ describes the motion of the particle in the absence of time dependent terms and is generally the Laplacian or some perturbation thereof, possibly discretized, the underlying Hilbert space being $\ell^{2}\left(\mathbb{Z}^{d}\right)$ or $L^{2}\left(\mathbb{R}^{d}\right)$. The time dependent term $\Lambda(t)$ is the operator of multiplication by a bounded function $\Lambda(x, t)$. Theorem 1 is relevant to the adiabatic evolution of an ensemble of non-interacting particles with Fermi statistics. The observables, in this case, are the Fermi-Dirac distributions $F_{\mu, \beta}\left(H_{o}\right)=\frac{1}{1+e^{\beta\left(H_{o}-\mu\right)}}$ at positive temperatures and the spectral projections $\chi\left(H_{o} \leq \mu\right)$ and/or $\chi\left(H_{o}<\mu\right)$ at zero temperature. We obtain an adiabatic evolution even if there is an eigenvalue at the chemical potential $\mu$ ! 
The heart of the matter lies in the proof of Theorem 1 under the additional assumption that $\Lambda$ is boundedly differentiable in norm, i.e., that

$$
\dot{\Lambda}(s):=\lim _{h \rightarrow 0} \frac{\Lambda(s+h)-\Lambda(s)}{h}
$$

exists in the norm topology for each $s \in(0,1)$ and $\sup _{s}\|\dot{\Lambda}(s)\|$ is finite. The extension to general $\Lambda$ is accomplished by a standard mollifier argument.

Specifically, we choose a positive smooth function $\phi(s)$ with compact support such that $\int \phi=1$ and set $\phi_{\epsilon}(s)=\epsilon^{-1} \phi(s / \epsilon)$. Then

$$
\Lambda_{\epsilon}(s)=\int \mathrm{d} s^{\prime} \phi_{\epsilon}\left(s-s^{\prime}\right) \Lambda\left(s^{\prime}\right)
$$

is boundedly differentiable and

$$
\lim _{\epsilon \rightarrow 0} \int_{0}^{1}\left\|\Lambda_{\epsilon}(s)-\Lambda(s)\right\|=0 .
$$

Let $U_{\tau, \epsilon}$ be the solution to the IVP (1.1) with $H_{\tau}(t / \tau)=H_{o}+(1 / \tau) \Lambda_{\epsilon}(t / \tau)$ and set $W_{\tau, \epsilon}(s)=U_{\tau}(\tau \cdot s)$. Then,

$$
\frac{\mathrm{d}}{\mathrm{d} s} W_{\tau, \epsilon}^{\dagger}(s) W_{\tau}(s)=W_{\tau, \epsilon}^{\dagger}(s)\left(\Lambda_{\epsilon}(s)-\Lambda(s)\right) W_{\tau}(s) .
$$

¿From this it follows that

$$
\left\|W_{\tau, \epsilon}(s)-W_{\tau}(s)\right\| \leq \int_{0}^{1} \mathrm{~d} s\left\|\Lambda_{\epsilon}(s)-\Lambda(s)\right\| \rightarrow 0,
$$

i.e., $W_{\tau, \epsilon}(s)$ converges to $W_{\tau}(s)$ uniformly in $s$ and $\tau$. By a standard "2- $\epsilon$ " argument the theorem now follows for $\Lambda$ in $L^{1}$ once it is verified for $\Lambda_{\epsilon}$. Hence, it suffices to show Theorem 1 for differentiable $\Lambda$.

Throughout the rest of the paper, $\Lambda$ will denote a uniformly bounded self-adjoint family which is differentiable in the norm topology with a uniformly bounded derivative $\dot{\Lambda}$.

The remainder of this section is devoted to the proof of those parts of Theorem 1 which follow from norm resolvent convergence. This part of the proof is very elementary but is also unrelated to the arguments in the subsequent sections. In section 3, we present a Lemma 1, which states that the portion of Theorem 1 related to functions of bounded variation $(B V)$ may be reduced to a statement about spectral projections. A proof of this Lemma, based on ideas that go back to Kato [11], is also given in section 3. In section 4 we prove Lemma 1 .

For a great many functions, $f$, the conclusion of Theorem 1 - i.e., eq. (2.2) follows from well known convergence theorems and a simple formula - eq. (2.10) which shows that

$$
\sup _{s \in[0,1]}\left\|W_{\tau}(s)\left(H_{o}-z\right)^{-1} W_{\tau}^{\dagger}(s)-\left(H_{o}-z\right)^{-1}\right\| \longrightarrow 0
$$

for every $z \notin \mathbb{R}$, which is to say that $W_{\tau}(s) H_{o} W_{\tau}^{\dagger}(s) \rightarrow H_{o}$ uniformly in $s$ in the "norm resolvent sense". The implications of norm resolvent convergence for Theorem 11 are that

(1) Eq. 2.2 holds in the norm topology for $f \in C_{o}$ [12, Thm. VIII.20]. 
(2) Eq. 2.2 holds in the strong operator topology for $f \in C_{b}$ or when $f$ is the characteristic function of an open interval $(a, b)$ provided that $a$ and $b$ are not eigenvalues of $H_{o}$. This follows from [12, Thm. VIII.20 and VIII.24] since "strong resolvent convergence" is implied by "norm resolvent convergence."

What is remarkable is that with some additional work we can prove eq. 2.2, for example, when $f$ is the characteristic function of an open interval $(a, b)$ and one or both of $a, b$ is an eigenvalue with arbitrary degeneracy.

To verify eq. (2.9), we use the identity

$$
\begin{aligned}
\left(H_{\tau}(s)-z\right)^{-1} & -W_{\tau}(s)\left(H_{\tau}(0)-z\right)^{-1} W_{\tau}(s)^{\dagger} \\
& =W_{\tau}(s) \int_{0}^{s} W_{\tau}(t)^{\dagger}\left(\frac{\mathrm{d}}{\mathrm{d} t}\left(H_{\tau}(t)-z\right)^{-1}\right) W_{\tau}(t) \mathrm{d} t W_{\tau}(s)^{\dagger},
\end{aligned}
$$

where $H_{\tau}(s)=H_{o}+\frac{1}{\tau} \Lambda(s)$. Eq. (2.10) follows from the fundamental theorem of calculus and the observation that

$$
\frac{\mathrm{d}}{\mathrm{d} t}\left(W_{\tau}(t)^{\dagger}\left(H_{\tau}(t)-z\right)^{-1} W_{\tau}(t)\right)=W_{\tau}(t)^{\dagger}\left(\frac{\mathrm{d}}{\mathrm{d} t}\left(H_{\tau}(t)-z\right)^{-1}\right) W_{\tau}(t) .
$$

Now, eq. (2.9) follows from eq. (2.10) because the latter implies that

$$
\left\|W_{\tau}(s)\left(H_{o}-z\right)^{-1} W_{\tau}(s)^{\dagger}-\left(H_{o}-z\right)^{-1}\right\| \leq \frac{C}{(\operatorname{Im} z)^{2}} \frac{1}{\tau},
$$

since

$$
\frac{\mathrm{d}}{\mathrm{d} t}\left(H_{\tau}(t)-z\right)^{-1}=\frac{1}{\tau}\left(H_{\tau}(t)-z\right)^{-1} \dot{\Lambda}(t)\left(H_{\tau}(t)-z\right)^{-1},
$$

and

$$
\left(H_{\tau}(s)-z\right)^{-1}=\left(H_{o}-z\right)^{-1}-\frac{1}{\tau}\left(H_{\tau}(s)-z\right)^{-1} \Lambda(s)\left(H_{o}-z\right)^{-1} .
$$

Before we proceed, let us describe an example which demonstrates that we cannot hope to prove eq. (2.2) for general $f$ in $B V$ using only the fact that $W_{\tau} H_{o} W_{\tau}^{\dagger}$ converges to $H_{o}$ in the norm resolvent sense. For this purpose it is sufficient to produce a sequence of unitary operators $V_{n}$ such that $V_{n} H_{o} V_{n}^{\dagger}$ converges to $H_{o}$ but nonetheless

$$
\underset{n \rightarrow \infty}{\operatorname{SOT}-\lim } V_{n} f\left(H_{o}\right) V_{n}^{\dagger} \neq f\left(H_{o}\right)
$$

for some function $f \in B V$.

For this purpose, consider the self adjoint operator on $\ell^{2}(\mathbb{Z})$ given in Dirac notation by $H_{o}=\sum_{m \neq 0} \frac{1}{m}|m\rangle\langle m|$, and for each $n$ let $V_{n}$ be the unitary on $\ell^{2}(\mathbb{Z})$ which "swaps 0 and $n$ ", i.e.,

$$
\left(V_{n} \psi\right)(m)=\left\{\begin{array}{ll}
\psi(m) & \text { if } m \neq 0, n \\
\psi(0) & \text { if } m=n \\
\psi(n) & \text { if } m=0
\end{array} .\right.
$$

Then $V_{n} H_{o} V_{n}^{\dagger}=H_{o}+\frac{1}{n}(|0\rangle\langle 0|-| n\rangle\langle n|)$. So $V_{n} H_{o} V_{n}^{\dagger} \rightarrow H_{o}$ in norm, and thus in norm resolvent sense. Yet,

$$
V_{n}|0\rangle\left\langle 0\left|V_{n}^{\dagger}=\right| n\right\rangle\langle n| \stackrel{\text { SOT }}{\longrightarrow} 0 \quad n \rightarrow \infty,
$$

and $|0\rangle\langle 0|=P_{0}$, the spectral projection of $H_{o}$ associated to eigenvalue 0 . 


\section{SOT CONVERGENCE FOR SPECTRAL PROJECTIONS}

The claim that eq. (2.2) holds whenever $f \in B V$ is, at heart, a statement about spectral projections as is indicated by the following lemma:

Lemma 1. Eq. (2.2) holds in the SOT for every $f \in B V$ if and only if it holds for all $f$ of the form $f(x)=\chi(x \leq E)$ or $f(x)=\chi(x \geq E)$ with any $E \in \mathbb{R}$.

We postpone the proof of Lemma 11 to section 4 and focus here on proving eq. 2.2 with $f(x)=\chi(x \geq E)$ and $f(x)=\chi(x \leq E)$ for every $E$ in $\mathbb{R}$.

In what follows we fix $E$ and take $P=\chi\left(H_{o} \leq E\right)$. The other case, $\chi\left(H_{o} \geq E\right)$, is handled in exactly the same way by changing $\leq$ to $\geq$ in the appropriate places. We must show that for any $\psi \in \mathcal{H}$

$$
\lim _{\tau \rightarrow \infty} \sup _{s \in[0,1]}\left\|\left(W_{\tau}(s) P W_{\tau}(s)^{\dagger}-P\right) \psi\right\|=0 .
$$

Our argument is stated most readily with the propagator $W_{\tau}(t, s)=W_{\tau}(t) W_{\tau}^{\dagger}(s)$ - note that $W_{\tau}(s) P W_{\tau}^{\dagger}(s)=W_{\tau}(s, 0) P W_{\tau}(0, s)$ and $P=W_{\tau}(s, s) P W_{\tau}(s, s)$. We would like to compare $W_{\tau}(s, t)$ with the propagator associated to $H_{o}$, so we define

$$
\Omega_{\tau}(t, s):=\mathrm{e}^{\mathrm{i} \tau(t-s) H_{o}} W_{\tau}(t) W_{\tau}(s)^{\dagger} .
$$

Since $\Omega_{\tau}(t, s)$ is unitary and the exponential of $H_{o}$ commutes with $P$ and

$$
\begin{aligned}
\left\|\left(W_{\tau}(s) P W_{\tau}(s)^{\dagger}-P\right) \psi\right\| & =\left\|\left(\Omega_{\tau}(0, s)^{\dagger} P \Omega_{\tau}(0, s)-P\right) \psi\right\| \\
& =\left\|\left[P, \Omega_{\tau}(0, s)\right] \psi\right\| .
\end{aligned}
$$

Finally, because $P$ is a projection

$$
\left[P, \Omega_{\tau}(t, s)\right]=P \Omega_{\tau}(t, s) \bar{P}-\bar{P} \Omega_{\tau}(t, s) P,
$$

where $\bar{P}=\mathbf{1}-P$. Therefore, eq. 3.1 will follow if we can verify that both terms on the right side of eq. (3.4) uniformly converge to zero in the SOT.

Consider the first term. Let $P_{\Delta}:=\chi\left(E<H_{0}<E+\Delta\right)$, then

$$
P \Omega_{\tau}(t, s) \bar{P}=P \Omega_{\tau}(t, s)\left(\bar{P}-P_{\Delta}\right)+P \Omega_{\tau}(t, s) P_{\Delta} .
$$

We will see below (Lemma 3) that the operator norm of $P \Omega\left(P-P_{\Delta}\right)$ is uniformly bounded by $1 / \tau \Delta$. Thus given $\psi \in \mathcal{H}$

$$
\left\|P \Omega_{\tau}(t, s) \bar{P} \psi\right\| \leq \frac{C}{\tau \Delta}\|\psi\|+\left\|P_{\Delta} \psi\right\| .
$$

If, for instance, $\Delta=1 / \sqrt{\tau}$ then both terms converges to zero since SOT-lim $P_{\Delta}=0$ - whether or not there is an eigenvalue at $E$.

The second term of (3.4) requires a little more care. Because $E$ may be an eigenvalue, we need to isolate the contribution from the associated projection $P_{E}=$ $\chi\left(H_{o}=E\right)$. Let $P_{\Delta}^{\prime}=\chi\left(E-\Delta<H_{o}<E\right)$ and consider

$$
\bar{P} \Omega_{\tau}(t, s) P=\bar{P} \Omega_{\tau}(t, s)\left(P-P_{\Delta}^{\prime}-P_{E}\right)+\bar{P} \Omega_{\tau}(t, s) P_{\Delta}^{\prime}+\bar{P} \Omega_{\tau}(t, s) P_{E} .
$$

As above, if we take $\Delta=1 / \sqrt{\tau}$ then the first and second terms tend uniformly to zero. That the third term also converges to zero is the content of the following lemma:

Lemma 2. Let $P_{E}:=\chi\left(H_{o}=E\right)$. Then $\left(\mathbf{1}-P_{E}\right) \Omega_{\tau}(t, s) P_{E}$ uniformly tends to zero in the strong operator topology. 
Proof. The operator $\Omega_{\tau}(t, s)$ satisfies a Volterra equation

$$
\Omega_{\tau}(t, s)=\mathbf{1}+\int_{s}^{t} \mathrm{~d} r K_{\tau}(r, s) \Omega_{\tau}(r, s)
$$

with

$$
K_{\tau}(r, s)=-\mathrm{ie}^{\mathrm{i} \tau(r-s) H_{o}} \Lambda(r) \mathrm{e}^{\mathrm{i} \tau(s-r) H_{o}} .
$$

By iterating eq. (3.8) we obtain a norm convergent series

$$
\Omega_{\tau}(t, s)=\sum_{n=0}^{\infty} A_{\tau}^{n}(t, s)
$$

where

$$
A_{\tau}^{n}(t, s)=\int_{s \leq r_{n} \leq \ldots \leq r_{1} \leq t} \ldots \int_{1} \mathrm{~d} r_{1} \ldots \mathrm{d} r_{n} K_{\tau}\left(r_{1}, s\right) \ldots K_{\tau}\left(r_{n}, s\right) .
$$

Since $A_{\tau}^{n}(t, s)$ is obtained by integrating a product of $n$ factors of $K$ over a simplex of volume $(t-s)^{n} / n$ ! we have the elementary norm bound

$$
\left\|A_{\tau}^{n}(t, s)\right\| \leq \frac{1}{n !} \kappa^{n}(t-s)^{n}
$$

where $\kappa=\sup _{r}\|\Lambda(r)\|$.

Using dominated convergence, we see from (3.10), (3.12), that it suffices to show for each $n$ that $\bar{P}_{E} A_{\tau}^{n}(t, s) P_{E} \rightarrow 0$ uniformly in the SOT. This may be proved as follows. First note that

$$
\begin{aligned}
& P_{E} K_{\tau}(r, s) P_{E}=-\mathrm{i} P_{E} \Lambda(r) P_{E}, \\
& \bar{P}_{E} K_{\tau}(r, s) P_{E}=-\mathrm{i} \bar{P}_{E} \mathrm{e}^{\mathrm{i} \tau(r-s)\left(H_{o}-E\right)} \Lambda(r) P_{E} .
\end{aligned}
$$

Next observe that

$$
\int_{s}^{r} \mathrm{~d} r^{\prime} \bar{P}_{E} \mathrm{e}^{\mathrm{i} \tau\left(r^{\prime}-s\right)\left(H_{o}-E\right)} \longrightarrow 0
$$

uniformly in the strong operator topology from which it follows via integration by parts that

$$
\int_{s}^{r} \mathrm{~d} r^{\prime} \bar{P}_{E} \mathrm{e}^{\mathrm{i} \tau\left(r^{\prime}-s\right)\left(H_{o}-E\right)} B\left(r^{\prime}\right) \longrightarrow 0
$$

for any differentiable family of operators $B(r)$ which does not depend on $\tau$.

Now consider the expression for $A_{\tau}^{n}$ obtained by inserting $\mathbf{1}=\bar{P}_{E}+P_{E}$ between the two right most factors of $K_{\tau}$ in the integral which appears in eq. (3.11). Proceed with the term obtained from $P_{E}$ by inserting $\bar{P}_{E}+P_{E}$ between the next two factors of $K$. Continue from right to left in this way, expanding only the terms obtained from $P_{E}$. We obtain an expression for $\bar{P}_{E} A_{\tau}^{n}(t, s) P_{E}$ as of sum of $n$ terms, the $j$ th term being

$$
\begin{aligned}
&(-\mathrm{i})^{j} \int_{s \leq r_{n} \leq \ldots \leq r_{1} \leq t} \ldots \int_{1} \mathrm{~d} r_{1} \ldots \mathrm{d} r_{n} \bar{P}_{E} K_{\tau}\left(r_{1}, s\right) \ldots K_{\tau}\left(r_{n-j}, s\right) \\
& \times \bar{P}_{E} \mathrm{e}^{\mathrm{i} \tau\left(r_{n-j+1}-s\right)\left(H_{o}-E\right)} \Lambda\left(r_{n-j+1}\right) P_{E} \ldots \Lambda\left(r_{n}\right) P_{E},
\end{aligned}
$$

which uniformly converges to zero by virtue of eq. (3.16). Since $A_{\tau}^{n}$ is a finite linear combination of terms which uniformly tend to zero it does so as well. 
It remains to show that $\left\|P \Omega_{\tau}(t, s)\left(\bar{P}-P_{\Delta}\right)\right\|$ is bounded by $1 / \tau \Delta$.

Lemma 3. Let $P_{1}:=\chi\left(H_{o} \leq E_{1}\right)$ and $P_{2}:=\chi\left(H_{o} \geq E_{2}\right)$ with $E_{2}>E_{1}$. Then

$$
\left\|P_{1} \Omega_{\tau}(t, s) P_{2}\right\| \leq \frac{C}{\Delta \tau},
$$

where $\Delta=E_{2}-E_{1}$ and $C$ is a constant which does not depend on $E_{1}$ or $E_{2}$. The same inequality holds with $P_{1}, P_{2}$ interchanged.

Proof. As in the proof of Lemma 2 the idea is to prove a bound on each term $A_{\tau}^{n}$ in the expansion for $\Omega_{\tau}$. In this case, we will show that

$$
\left\|P_{1} A_{\tau}^{n}(s, t) P_{2}\right\| \leq \frac{n}{\tau \Delta} \frac{\alpha^{n}}{(n-1) !}
$$

where $\alpha$ is a constant independent of $s, t$. Summing these bounds clearly implies eq. (3.18) - see eq. (3.10).

The main step is to show that

$$
\left\|P_{1} \int_{t}^{s} d r K_{\tau}(r, s) P_{2}\right\| \leq \frac{C}{\Delta \tau},
$$

and the same with $P_{1}$ and $P_{2}$ interchanged. The idea is that, since $K_{\tau}(r, s)=$ $\mathrm{e}^{\mathrm{i} \tau(r-s) H_{o}} \Lambda(r) \mathrm{e}^{\mathrm{i} \tau(s-t) H_{o}}$ and the spectral supports of $P_{1}$ and $P_{2}$ are distance $\Delta$ apart, the integral over $r$ has a highly oscillating phase of order $\tau \Delta$. For a rigorous argument, however, it is convenient to use a commutator equation and integration by parts to extract eq (3.20). This method goes back to Kato [11].

The commutator $\left[H_{o}, X\right]$ might be ill defined if $H_{o}$ is unbounded. Thus we introduce a cutoff and work instead with $\left[H_{o}, P_{M} X P_{M}\right]$ where $P_{M}=\chi(-M<$ $\left.H_{o}<M\right)$ and $M \in(0, \infty)$. At the end of the argument we take $M \rightarrow \infty$. The $X$ we have in mind is

$$
X(r):=\frac{1}{2 \pi \mathrm{i}} \int_{\Gamma} \mathrm{d} z P_{1} R(z) \Lambda(r) R(z) P_{2} .
$$

where $R(z):=\left(H_{0}-z\right)^{-1}$ and the contour $\Gamma$ is the line $\left\{E^{\prime}+\mathrm{i} \eta: \eta \in \mathbb{R}\right\}$ with $E^{\prime}=\left(E_{2}+E_{1}\right) / 2$. A simple calculation yields

$$
\left[H_{o}, P_{M} X(r) P_{M}\right]=P_{M} P_{1} \Lambda(r) P_{2} P_{M}
$$

Therefore

$$
\begin{aligned}
P_{M} P_{1} K_{\tau}(r, s) P_{2} P_{M}= & {\left[H_{o}, \mathrm{e}^{\mathrm{i} \tau(r-s) H_{o}} P_{M} X(r) P_{M} \mathrm{e}^{\mathrm{i} \tau(s-r) H_{o}}\right] } \\
=\frac{1}{\mathrm{i} \tau}\left(\frac{\mathrm{d}}{\mathrm{d} r}\left(P_{M} \mathrm{e}^{\mathrm{i} \tau(r-s) H_{o}} X(r) \mathrm{e}^{\mathrm{i} \tau(s-r) H_{o}} P_{M}\right)\right. & \left.\quad-P_{M} \mathrm{e}^{\mathrm{i} \tau(r-s) H_{o}} \dot{X}(r) \mathrm{e}^{\mathrm{i} \tau(s-r) H_{o}} P_{M}\right) .
\end{aligned}
$$

However $X(r)$ and $\dot{X}(r)$ are uniformly bounded, $\|X(r)\|,\|\dot{X}(r)\| \leq C / \Delta$, so integrating (3.23) yields

$$
\left\|P_{M} P_{1} \int_{t}^{s} d r K_{\tau}(r, s) P_{2} P_{M}\right\| \leq \frac{C}{\Delta \tau} .
$$

In the limit $M \rightarrow \infty$ this implies eq. 3.20 by lower semi-continuity of the norm. The second case with $P_{1}$ and $P_{2}$ interchanged follows with an obvious modification of $X$. 
The rest of the argument is similar to the proof of Lemma 2. We insert a decomposition of the identity $\mathbf{1}=Q+\bar{Q}$ between the factors of $K$ in the integral expression for $A_{\tau}^{n}$, eq. (3.11). To apply 3.20 ), we should maintain a spectral gap between the projections which sits to the left and right of $K$. Therefore we define $Q_{j}:=\chi\left(H_{0} \leq Q_{1}+j / n \Delta\right)$ for $j=0, \ldots, n$ and insert $1=Q_{j}+\bar{Q}_{j}$ between the $j$ th and $(j+1)$ th factors of $K$. With these insertions, $P_{1} A_{\tau}^{n} P_{2}$ breaks into $2^{n}$ terms and each term includes at least one factor of the type $Q_{j} K_{\tau}\left(r_{j+1}, s\right) \bar{Q}_{j+1}$ or $\bar{Q}_{j} K_{\tau}\left(r_{j+1}, s\right) Q_{j+1}$ where there is a gap of size $\Delta / n$ between the spectral supports of the two projections. We apply integration by parts to the integral over $r_{j+1}$ to obtain a factor which may be bounded by eq. (3.20):

$$
\begin{aligned}
\int_{0}^{r_{j}} \mathrm{~d} r_{j+1} Q_{j} K_{\tau}\left(r_{j+1}, s\right) & \bar{Q}_{j+1} B\left(r_{j+1}\right) \\
= & \int_{0}^{r_{j}} \mathrm{~d} r_{j+1} Q_{j} \int_{r^{\prime}}^{r_{j}} \mathrm{~d} r^{\prime} K_{\tau}\left(r^{\prime}, s\right) \bar{Q}_{j+1} \dot{B}\left(r_{j+1}\right) .
\end{aligned}
$$

Elementary norm estimates and eq (3.20) now show that each of the $2^{n}$ terms is bounded by $n \beta^{n} /(\Delta \tau(n-1)$ !) for some $\beta$ which implies eq. (3.19) with $\alpha=2 \beta$.

\section{Integration BY PARTS AND THE PROOF OF LEMMA 1}

Turning to the proof of Lemma 1, we note that the spectral theorem provides the representation

$$
f\left(H_{o}\right)=\int f(E) \mathrm{d} P_{o}(E)
$$

valid for bounded measurable $f$. The goal is to integrate this expression by parts thereby obtaining an expression involving $\mathrm{d} f$ and $P_{o}(E)=\chi\left(H_{o} \leq E\right)$. This argument works precisely when $f \in B V$ as we shall now explain.

The projection valued measure $\mathrm{d} P_{o}(E)$ is the differential of $P_{o}(E)=\chi\left(H_{o} \leq E\right)$ which is of bounded variation in the strong operator topology. That is, for any $\psi \in \mathcal{H}$,

$$
\sup _{n \geq 1} \sup _{E_{0}<\cdots<E_{n} \in \mathbb{R}} \sum_{j=1}^{n}\left\|P_{o}\left(E_{j}\right) \psi-P_{o}\left(E_{j-1}\right) \psi\right\|<\infty .
$$

We could equally well work with $P_{o}(E)=\chi\left(H_{o}<E\right)$ or a number of other choices - the distinction being meaningful only if $H_{o}$ has point spectrum.

Since the function $P_{o}$ is SOT-continuous from the left at every $E$, i.e. $P_{o}(E-$ $0)=P_{o}(E)$, we may integrate (4.1) by parts whenever $f \in B V$ and everywhere continuous from the right:f

$$
f\left(H_{o}\right)=f(\infty) \mathbf{1}-\int \mathrm{d} f(E) P_{o}(E)
$$

$f \in B V$ and continuous from the right.

\footnotetext{
${ }^{b}$ The extension of integration by parts to functions in $B V$ is a standard part of real analysis we direct the reader to 10, Ch. 3] for details.
} 
For general $f \in B V$ this formula is replaced by

$$
\begin{aligned}
f\left(H_{o}\right)=f(\infty) \mathbf{1} & -\int \mathrm{d} f(E) \chi\left(H_{o} \leq E\right) \\
& +\sum_{E \in \mathbb{R}}(f(E)-f(E+0)) \chi\left(H_{o}=E\right) .
\end{aligned}
$$

Note that $\sum_{E \in \mathbb{R}}|f(E)-f(E+0)| \leq \operatorname{Var}(f)<\infty$. In particular, there can be only countably many $E \in \mathbb{R}$ for which $f(E) \neq f(E+0)$.

Now suppose that

$$
\text { SOT-lim } W_{\tau}(s) A W_{\tau}(s)^{\dagger}=A
$$

uniformly in $s$ whenever $A=\chi\left(H_{o} \leq E\right)$ or $A=\chi\left(H_{o} \geq E\right)$ with $E \in \mathbb{R}$. Since

$$
\chi\left(H_{o}=E\right)=\chi\left(H_{o} \leq E\right)+\chi\left(H_{o} \geq E\right)-\mathbf{1},
$$

eq. (4.5) also holds with $A=\chi\left(H_{o}=E\right)$. Now given $f \in B V$, use (4.4) to express $f\left(H_{o}\right)$ and find that

$$
\mathrm{SOT}-\lim W_{\tau}(s) f\left(H_{o}\right) W_{\tau}(s)^{\dagger}=f\left(H_{o}\right)
$$

uniformly in $s$ by dominated convergence.

\section{Why the NORM TOPOlOGY IS INADEQUATE - AN EXAMPLE}

The following example, due to Michael Aizenman, is motivated by the consideration of systems with dense point spectrum.

We begin with a comment which, although mathematically trivial, already contains a key observation. If a family of operators, $A_{\tau}$, converges (as $\tau \rightarrow \infty$ ) in the strong operator topology then although for any vector $\psi$ the family of vectors $A_{\tau} \psi$ is convergent - this is the very definition of SOT convergence - nothing can be said regarding the rate of convergence. In fact the essential difference with norm convergence is that in the norm case the vectors $A_{\tau} \psi$ all converge at the same rate.

For a specific example, consider the countable collection of non interacting twolevel systems each perturbed by a weak perturbation (strength $\sim 1 / \tau$ ):

$$
H_{\tau}=H_{o}+\frac{1}{\tau} \Lambda, \quad H_{o}=\sum_{k=0}^{\infty} \oplus m_{k} \sigma_{z}, \quad \Lambda=\sum_{k=0}^{\infty} \oplus \sigma_{x},
$$

with $\sigma_{z}, \sigma_{x}$ the Pauli spin matrices and $m_{k}=1 / k$. The perturbation $\Lambda$ is time independent, but still Theorem 1 applies. Of course, the unitary evolution $U_{\tau}$ associated with Hamiltonian $H_{\tau}$ decomposes into a direct sum of two by two matrices $U_{\tau}^{k}$ each generated by $H_{k, \tau}=(1 / k) \sigma_{z}+(1 / \tau) \sigma_{x}$.

Let us choose $f\left(H_{o}\right)=P_{o}$ to be the spectral projection onto negative energies: $P_{o}=\chi\left(H_{o}<0\right)$. We will show,

$$
\limsup _{\tau \rightarrow \infty}\left\|U_{\tau}(\tau s) P_{o} U_{\tau}^{\dagger}(\tau s)-P_{o}\right\| \geq \alpha(s)
$$

where $\alpha(s)>0$ for every $s \in(0,1)$, although, in accordance with Theorem 11,

$$
\text { SOT-lim } U_{\tau}(\tau s) P_{o} U_{\tau}^{\dagger}(\tau s)=P_{o} .
$$

Indeed, consider the particular sequence $\tau_{n}:=n$. For each $n$, the evolution of the two-level system with $m_{n}:=1 / n$ obeys

$$
\mathrm{i} \dot{U}_{\tau_{n}}^{n}(t)=\frac{1}{n}\left(\sigma_{z}+\sigma_{x}\right) U_{\tau_{n}}^{n}(t)
$$


Thus, the matrix $V(s):=U_{\tau_{n}}^{n}\left(\tau_{n} s\right)$ is independent of $n$ and may be obtained by integrating

$$
\mathrm{i} \dot{V}(s)=\left(\sigma_{z}+\sigma_{x}\right) V(s)
$$

with initial condition $V(0)=\left(\begin{array}{ll}1 & 0 \\ 0 & 1\end{array}\right)$. It is now a simple matter to check that

$$
\left\|V(s)\left(\begin{array}{ll}
0 & 0 \\
0 & 1
\end{array}\right) V(s)^{\dagger}-\left(\begin{array}{ll}
0 & 0 \\
0 & 1
\end{array}\right)\right\|>0
$$

for all $s \in(0,1)$. Since, as mentioned above, $U_{\tau}$ is the direct sum of the two by two matrices $U_{\tau}^{k}$ we have

$$
\left.\| U_{\tau_{n}}\left(\tau_{n} s\right) P_{o} U_{\tau_{n}}^{\dagger}\left(\tau_{n} s\right)\right)-P_{o}\|\geq\| V(s)\left(\begin{array}{cc}
0 & 0 \\
0 & 1
\end{array}\right) V(s)^{\dagger}-\left(\begin{array}{cc}
0 & 0 \\
0 & 1
\end{array}\right) \|>0 .
$$

\section{The SChröDinger PiCture - A THEOREM AND A COUnter-EXAMPle}

Theorem 11 describes the adiabatic limit of the Heisenberg picture of quantum dynamics. As for the Schrödinger picture, there is no reason to expect $U_{\tau}(\tau s)$ to converge to anything at all, since even the unperturbed evolution, $\mathrm{e}^{-\mathrm{i} \tau s H_{o}}$, does not have a large $\tau$ limit.

With this in mind, it is natural to ask whether, in some sense, $U_{\tau}$ is asymptotically equal to $\mathrm{e}^{-\mathrm{i} \tau s H_{o}}$. To test this idea we consider the evolution

$$
\Omega_{\tau}(s):=\mathrm{e}^{\mathrm{i} \tau s H_{o}} U_{\tau}(\tau s)
$$

which represents, physically, a process in which the system is evolved forward in time according to the perturbed dynamics and then backwards in time according to the unperturbed dynamics.

If $H_{o}$ admits an eigenfunction decomposition, i. e., if the spectrum of $H_{o}$ is pure point, then a simple extension of the proof of Lemma 2 shows that $\Omega_{\infty}$ does exist and even allows us to calculate it.

Theorem 2. Let $H_{O}$ be a self-adjoint operator with only pure point spectrum. It $U_{\tau}$ satisfies the initial problem (1.1) with $H_{\tau}(t / \tau)=H_{o}+(1 / \tau) \Lambda(t / \tau)$ where $\Lambda(\cdot)$ is a self-adjoint family which is $L^{1}$ in norm, then

$$
\underset{\tau \rightarrow \infty}{\operatorname{SOT}-l i m} \mathrm{e}^{\mathrm{i} \tau s H_{o}} U_{\tau}(\tau s)=\Omega_{\infty}(s)
$$

where $\Omega_{\infty}(s)$ is the unitary operator which commutes with $H_{o}$ and satisfies the initial value problem

$$
\left\{\begin{array}{l}
\mathrm{i} \dot{\Omega}_{\infty}(s)=\left(\sum_{E \in \sigma\left(H_{o}\right)} P_{E} \Lambda(s) P_{E}\right) \Omega_{\infty}(s) \\
\Omega_{\infty}(0)=\mathbf{1}
\end{array}\right.
$$

where $P_{E}=\chi\left(H_{o}=E\right)$ is the orthogonal projection onto the space of eigenvectors of $H_{o}$ with eigenvalue $E$.

Remarks:

(1) When there is a uniform lower bound on the spacing between neighboring eigenvalues, a classical result of Born and Fock [1] shows that the convergence occurs in the norm topology. 
(2) This theorem is of particular interest if $H_{o}$ has only dense point spectrum as is true of discrete random Schrödinger operators in the large disorder regime - see [13] for one perspective on this subject.

Proof. In the notation of Sec. 3, the evolution considered here $-\Omega_{\tau}(s)-$ is equal to the propagator $\Omega_{\tau}(s, 0)$. Thus, by lemma 2, we see that $\left(\mathbf{1}-P_{E}\right) \Omega_{\tau}(s) P_{E}$ converges to zero uniformly in the strong operator topology for every $E$. To complete the proof of Theorem 2, we let $\Omega_{\infty}(s)$ denote the solution to the initial value problem (6.3) and show that

$$
\mathrm{SOT}-\lim P_{E} \Omega_{\tau}(s) P_{E}=P_{E} \Omega_{\infty}(s) P_{E}
$$

for each $E \in \sigma\left(H_{o}\right)$.

As we saw in the proof of Lemma $2, \Omega_{\tau}(s)$ satisfies a Volterra-type equation:

$$
\Omega_{\tau}(s)=-\mathrm{i} \int_{0}^{s} \mathrm{~d} r K_{\tau}(r) \Omega_{\tau}(r),
$$

with $K_{\tau}(r)=-\mathrm{ie}^{\mathrm{i} \tau r H_{o}} \Lambda(r) \mathrm{e}^{-\mathrm{i} \tau r H_{o}}$. Inserting into this expression $\mathbf{1}=P_{E}+\left(\mathbf{1}-P_{E}\right)$ between $K_{\tau}$ and $\Omega_{\tau}$ we obtain

$$
\begin{aligned}
P_{E} \Omega_{\tau}(s) P_{E}=P_{E}-\mathrm{i} \int_{0}^{s} \mathrm{~d} r P_{E} \Lambda & (r) P_{E} \Omega_{\tau}(r) P_{E} \\
& -\mathrm{i} \int_{0}^{s} \mathrm{~d} r P_{E} K_{\tau}(r)\left(\mathbf{1}-P_{E}\right) \Omega_{\tau}(r) P_{E},
\end{aligned}
$$

since $P_{E} K_{\tau}(r) P_{E}=\Lambda(r)$. The last term on the right side converges to zero uniformly in the strong operator topology, again by Lemma 2 .

It is clear from eq. (6.6) that if the limit of $P_{E} \Omega_{\tau}(s) P_{E}$ exists, then it obeys the evolution equation (6.3). However, eq. (6.6) does not directly imply that the limit exists. On the other hand, $\Omega_{\infty}$ also satisfies a Volterra-type equation which when subtracted from (6.6) yields, for the difference $\mathcal{E}_{\tau}(s)=P_{E} \Omega_{\tau}(s) P_{E}-P_{E} \Omega_{\infty}(s) P_{E}$,

$$
\mathcal{E}_{\tau}(s)=-\mathrm{i} \int_{0}^{s} \mathrm{~d} r P_{E} \Lambda(r) P_{E} \mathcal{E}_{\tau}(r)+R_{\tau}(s),
$$

where the remainder $R_{\tau}(s)$ is the last term of $(6.6)$ and converges to zero uniformly in the strong operator topology. Using Gronwall's lemma [14], we conclude from (6.7) that $\mathcal{E}_{\tau}(s)$ converges to zero uniformly in the strong operator topology.

More concretely, let $\psi$ be any vector. Then (6.7) yields

$$
\left\|\mathcal{E}_{\tau}(s) \psi\right\| \leq \int_{0}^{s} \mathrm{~d} r\left\|P_{E} \Lambda(r) P_{E}\right\|\left\|\mathcal{E}_{\tau}(r) \psi\right\|+\left\|R_{\tau}(s) \psi\right\| .
$$

¿From this together with the classical Gronwall lemma we learn that

$$
\left\|\mathcal{E}_{\tau}(s) \psi\right\| \leq\left[\sup _{s}\left\|R_{\tau}(s) \psi\right\|\right] \mathrm{e}^{\int_{0}^{1}\|\Lambda(r)\| \mathrm{d} r} .
$$

Since the factor in brackets converges to zero as $\tau \rightarrow \infty$ so does the right hand side of the above inequality.

We conclude with an example which shows that in general $\Omega_{\tau}(s)$ need not have a limit. Take $H_{o}$ to be differentiation, id $/ \mathrm{d} x$, on $L^{2}(\mathbb{R})$ and let $\Lambda(s)$ be the operator of multiplication by a function $\Lambda(x, s)$. Since $\mathrm{e}^{-\mathrm{i} t H_{o}}$ is a shift by $t$, the generator 
of $K_{\tau}(s)$ is the operator of multiplication by $-\mathrm{i} \Lambda(x-\tau s, s)$. Thus, since $K_{\tau}(s)$ is a commuting family,

$$
\Omega_{\tau}(s)=\mathrm{e}^{-\mathrm{i} \int_{0}^{s} \mathrm{~d} r \Lambda(x-\tau r, r)} .
$$

If, for instance, $\Lambda(x, r)$ is the indicator function of the set $\cup_{n}\left[2^{2 n}, 2^{2 n+1}\right]$ then the right hand side has no limit as $\tau \rightarrow \infty$.

In light of this example, it is interesting to ask what conditions may be placed on $\Lambda(r)$ to ensure the convergence of $\Omega_{\tau}(s)$. This question is similar to the problem of existence of the wave operators which arises in scattering theory, and can be dealt accordingly.

Acknowledgments. We are grateful to M. Aizenman for the example presented in Section 5 as well as the suggestion to consider the strong operator topology. We are also indebted to Y. Avron for the insight that the norm adiabatic theorem is linked with the presence of an intrinsic time scale. This work was partially supported by the NSF Grant PHY-9971149 (AE). 


\section{REFERENCES}

[1] M. Born and V. Fock. Beweis des adiabatensatzes. Z. Phys., 51, 165-180, 1928.

[2] J. E. Avron, R. Seiler, and L. G. Yaffe. Adiabatic theorems and applications to the quantum Hall effect. Comm. Math. Phys., 110, 33-49, 1987.

[3] G. Nenciu. On the adiabatic theorem of quantum mechanics. J. Phys. A, 13, L15-L18, 1980.

[4] G. A. Hagedorn. Adiabatic expansions near eigenvalue crossings. Ann. Physics, 196, 278-295, 1989.

[5] A. Joye, F. Monti, S. Guérin, and H. R. Jauslin. Adiabatic evolution for systems with infinitely many eigenvalue crossings. J. Math. Phys., 40, 5456-5472, 1999.

[6] J. E. Avron, J. S. Howland, and B. Simon. Adiabatic theorems for dense point spectra. Comm. Math. Phys., 128, 497-507, 1990.

[7] F. Bornemann. Homogenization in time of singularly perturbed mechanical systems. SpringerVerlag, Berlin, 1998.

[8] J. E. Avron and A. Elgart. Adiabatic theorem without a gap condition. Comm. Math. Phys., 203:445-463, 1999.

[9] A. Joye and Ch.-Ed. Pfister. Exponential estimates in adiabatic quantum evolution. In Proceedings of the XIIth International Congress of Mathematical Physics, Brisbane, 1997.

[10] G. B. Folland. Real analysis. John Wiley \& Sons Inc., New York, 1984. Modern techniques and their applications, A Wiley-Interscience Publication.

[11] T. Kato. On the adiabatic theorem of quantum mechanics. Phys. Soc. Jap., 5, 435-9, 1958.

[12] M. Reed and B. Simon. Methods of modern mathematical physics. I. Academic Press Inc. [Harcourt Brace Jovanovich Publishers], New York, second edition, 1980. Functional analysis.

[13] M. Aizenman and S. Molchanov. Localization at large disorder and at extreme energies: an elementary derivation. Comm. Math. Phys., 157, 245-278, 1993.

[14] T. H. Gronwall. Note on the derivatives with respect to a parameter of the solutions of a system of differential equations. Ann. Math., 20, 292-296, 1919. 